\title{
Exposure to aflatoxins and fumonisins and linear growth of children in rural Ethiopia: a longitudinal study
}

\author{
Masresha Tessema ${ }^{1,2,3, *}$ (1), Hugo De Groote ${ }^{4}$, Inge D Brouwer ${ }^{1}$, Marthe De Boevre ${ }^{5}$, \\ Arnau Vidal Corominas ${ }^{5}$, Barbara J Stoecker ${ }^{6}$, Edith JM Feskens ${ }^{1}$, Tefera Belachew ${ }^{3}$, \\ Anastasia Karakitsou ${ }^{1}$ and Nilupa S Gunaratna ${ }^{7}$ \\ 'Division of Human Nutrition and Health, Wageningen University \& Research, Wageningen, The Netherlands: \\ ${ }^{2}$ Ethiopian Public Health Institute, Addis Ababa, Ethiopia: ${ }^{3}$ Human Nutrition Unit, Jimma University, Jimma, Ethiopia: \\ ${ }^{4}$ International Maize and Wheat Improvement Centre, Nairobi, Kenya: ${ }^{5}$ Centre of Excellence in Mycotoxicology and \\ Public Health, Faculty of Pharmaceutical Sciences, Ghent University, Gent, Belgium: ${ }^{6}$ Department of Nutritional \\ Sciences, Oklahoma State University, Stillwater, OK, USA: ${ }^{7}$ Department of Public Health, Purdue University, \\ West Lafayette, IN, USA
}

Submitted 12 December 2019: Final revision received 21 December 2020: Accepted 18 January 2021: First published online 1 February 2021

\begin{abstract}
Objective: We hypothesise that exposure to aflatoxins and fumonisins, measured in serum, alters protein synthesis, reducing serum protein and insulin-like growth factor 1 (IGF-1), increasing inflammation and infection, leading to child's linear growth failure.

Design: Children 6-35 months, stratified by baseline stunting, were subsampled from an intervention trial on quality protein maize consumption and evaluated at two time-points.

Setting: Blood samples and anthropometric data were collected in the pre-harvest (August-September 2015) and post-harvest (February 2016) seasons in rural Ethiopia.

Participants: 102 children (50 stunted and 52 non-stunted).

Results: Proportions of children exposed to aflatoxin G1, aflatoxin G2 and aflatoxin M1 were higher in the pre-harvest (8, 33 and 7, respectively) compared to post-harvest season (4, 28 and 4, respectively). The proportion of children exposed to any aflatoxin was higher in the pre-harvest than post-harvest season $(51 \% v$. $41 \%$ ). Fumonisin exposure ranged from $0 \%$ to $11 \%$. In joint statistical tests, aflatoxin exposure was associated with serum biomarkers of inflammation (C-reactive protein, $\alpha$-1-glycoprotein) and protein status (transthyretin, lysine, tryptophan), IGF-1 and linear growth (all $P<0 \cdot 01$ ). However, exposure to specific aflatoxins was not significantly associated with any biomarkers or outcomes (all $P>0 \cdot 05$ ).

Conclusions: Aflatoxin exposure among rural Ethiopian children was high, with large variation between seasons and individual aflatoxins. Fumonisin exposure was low. There was no clear association between aflatoxin exposure and protein status, inflammation or linear growth. A larger study may be needed to examine the potential biological interactions, and the assessment of aflatoxins in food is needed to determine sources of high exposure.
\end{abstract}

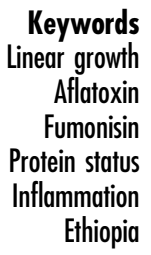

Keywords Aflatoxin Fumonisin Protein status Ethiopia
Poor linear growth of children, manifested as stunting, is the most prevalent form of undernutrition globally and has been associated with adverse health outcomes ${ }^{(1)}$. Despite the international commitment to reduce the number of stunted children under 5 years by $40 \%$ by $2030^{(2,3)}$, current nutritional interventions alone are unlikely to meet this target ${ }^{(4)}$. The aetiology of linear growth failure is poorly understood, which has hampered the development of effective interventions ${ }^{(5,6)}$.

Many children in developing countries are not only stunted but also chronically exposed to mycotoxins - toxic fungal metabolites such as aflatoxins and fumonisins ${ }^{(7)}$. 
Both are common contaminants of maize and maize-based products in tropical countries ${ }^{(8)}$. Another major source of exposure to aflatoxins is through the consumption of groundnuts $^{(9)}$. Aflatoxins are produced by Aspergillus flavus and A.parasiticus on different crops, both in the field and in storage ${ }^{(10)}$. Aflatoxins are the most potent genotoxic and carcinogenic mycotoxins ${ }^{(11)}$, and exposure to aflatoxins, mainly aflatoxin B1 (AFB1), aflatoxin B2 (AFB2), aflatoxin G1 (AFG1) and aflatoxin G2 (AFG2), can cause hepatocellular cancer ${ }^{(12)}$. Exposure to multiple aflatoxins measured in serum is poorly studied. Fumonisins are produced mainly by Fusarium verticillioides (Sacc.) Nirenberg and F. proliferatum (Matsush.) ${ }^{(10)}$. Contamination of children's complementary food with fumonisins has been reported in some countries ${ }^{(8,13-16)}$; however, the presence of fumonisins in children's serum has not been studied.

Based on associations in observational studies in humans, there is a growing concern that exposure to aflatoxins is associated with impaired linear growth of children in developing countries ${ }^{(17-22)}$. However, research findings are inconsistent, and the mechanisms are not clear. Observational studies in West Africa (Benin, Gambia and Nigeria) found an inverse association between AFB1 exposure and linear growth of children as measured by heightfor-age $Z$-score $(H A Z)^{(17-22)}$. Likewise, a study from Mexico showed that aflatoxin exposure, measured using aflatoxin B1-lysine (AFB1-Lys), was inversely associated with linear growth $^{(18)}$. In contrast, studies in East Africa ${ }^{(23-25)}$ and Nepal $^{(26)}$ did not find significant association between aflatoxin exposure (measured by aflatoxin-albumin or AFB1-Lys per milligram albumin) and linear growth of children. A recent intervention study among Kenyan children suggested that improving household access to aflatoxin-free maize reduced aflatoxin biomarker concentration in serum, but showed no effect on children's linear growth ${ }^{(23)}$.
The biological mechanisms through which aflatoxin exposure affects the linear growth of children are not clear. It has been proposed that exposure to aflatoxins may inhibit the synthesis of proteins, including insulin-like growth factor 1 (IGF-1), or that it may increase inflammation and risk of infection. Each of these effects could in turn reduce child growth ${ }^{(27)}$. However, these hypotheses have not been formally investigated to date in humans. Therefore, we assessed children's exposure to aflatoxins and fumonisins, as measured in serum, in both pre-harvest and post-harvest seasons. Given the high prevalence of aflatoxin exposure, we then investigated associations between aflatoxin exposure and biomarkers of inflammation and protein status as well as linear growth.

\section{Methods}

\section{Conceptual framework}

We hypothesised that exposure to aflatoxins could lead to linear growth failure either by inhibiting protein synthesis or by increasing inflammation or incidence of infection (Fig. 1). Household wealth and child's demographic characteristics, such as sex and age, could influence both exposure to aflatoxins and linear growth.

\section{Study setting}

The study used a subsample of children aged 6-35 months, stratified by baseline stunting, from an intervention trial on the consumption of quality protein maize (QPM) in rural Ethiopia (ClinicalTrials.gov identifier NCT02710760 and AEA RCT Registry $\# 0000786)^{(28)}$, where maize is the predominant staple used in children's complementary foods. QPM varieties have improved nutritional quality but are

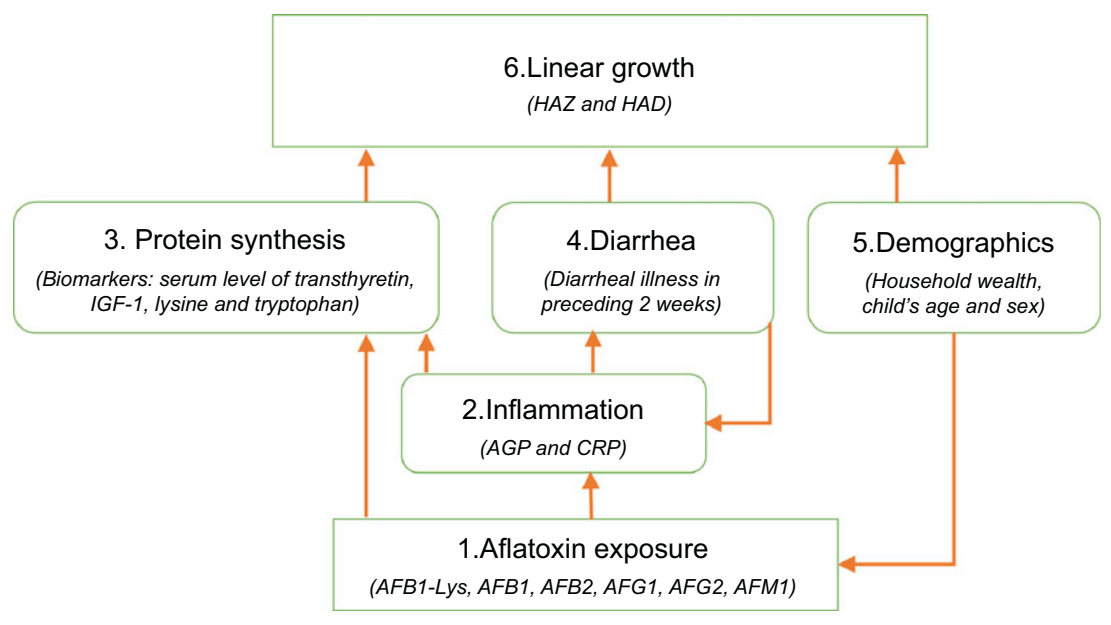

Fig. 1 Conceptual framework: (1) Aflatoxins exposure was measured by the biomarkers AFB1 lysine, AFB1, AFB2, AFG1, AFG2 and AFM1 from serum samples; (2) Inflammation was measured by inflammation biomarkers: C-reactive protein (CRP) and $\alpha$-1-glycoprotein protein (AGP) concentration from serum samples; (3) Protein synthesis was measured by protein and amino acid biomarkers in serum: Insulin-like growth factor 1 (IGF-1), transthyretin, lysine and tryptophan; (4) Diarrhea was measured using the mother's/ female caregiver's recall over the preceding two weeks; (5) Wealth tertiles were constructed based on household assets (primarily agricultural transportation and household implements) using principal component analysis (PCA); (6) Linear growth was measured using height-for-age Z-score (HAZ) and height-for-age difference (HAD) 
susceptible to mycotoxin contamination, as are all other maize varieties. In total, the trial enrolled 873 children. These children were stratified by baseline stunting status, and a random subsample of children was selected in each stratum (fifty stunted and fifty-two non-stunted for a total of 102 children). The objective of the trial was to assess the effect of promoting adoption and consumption of QPM on protein status and linear growth of children. The study protocol and sample characteristics are described elsewhere $^{(28,29)}$. Children were eligible for inclusion in the overall trial if they met the following criteria: (1) the household had at least one child aged 6-35 months at recruitment in July-September 2015; (2) the household had at least one member who had attended an agricultural demonstration conducted by the Nutritious Maize for Ethiopia (NuME) project in November 2014-January 2015; and (3) the household provided informed consent to participate in the study.

\section{Data collection}

Data were collected at three time-points: baseline (pre-harvest season, August-September 2015), midline (post-harvest season, February 2016) and endline (JuneAugust 2016). Questionnaires were administered to the household head at baseline and midline, and to the primary caregiver at all three time-points. Topics in the caregiver surveys included demographics, household roster (baseline only) and child's health and illness. Topics in the household head surveys included demographics and household assets. Anthropometrics (i.e. height/length along with sex and age) were collected from all children at all three time-points following standard measurement procedures $^{(30)}$. As mycotoxin exposure, based on serum levels, was only assessed at baseline and midline, this study focuses on questionnaires and anthropometric data from these two time-points. Diarrheal illness was assessed by asking the primary caregiver whether in the preceding 2 weeks the child had diarrhoea ( $\geq 3$ loose or watery motions in a 24-h period).

Venous blood $(5 \mathrm{ml})$ was collected in the pre-harvest (baseline) and post-harvest (midline) seasons by trained phlebotomists using trace element-free collection tubes (Vacutainer, Becton Dickenson). A temporary field laboratory was set up for the laboratory technologist to centrifuge and aliquot the serum immediately into appropriate cryovials. All samples for laboratory analysis were transported promptly after collection in cold boxes containing frozen gel packs $\left(-20^{\circ} \mathrm{C}\right)$ and stored at $-80{ }^{\circ} \mathrm{C}$ prior to analysis.

\section{Laboratory analyses}

Aflatoxins (AFB1-Lys, AFB1, AFB2, AFG1, AFG2 and AFM1) and fumonisins (hydrolysed fumonisin B1, fumonisin B1, fumonisin B2 and fumonisin B3) were analysed with liquid chromatography-tandem MS
(LC-MS/MS) ${ }^{(31)}$. Isotope-labelled ${ }^{13} \mathrm{C}_{17}$ aflatoxin was used as the internal standard. A protein precipitation method with few variations was used to prepare samples for analysis $^{(31)}$. Details about materials and reagents used for the analyses of aflatoxins and fumonisins and about sample preparation are described in supplementary material. The analyses of serum transthyretin, IGF-1, C-reactive protein (CRP) and $\alpha$-1-glycoprotein concentration (AGP) were described by Tessema et $a l .{ }^{(29)}$. Serum transthyretin, IGF-1, AGP and CRP were analysed at the Ethiopian Public Health Institute laboratory, certified by the Ethiopian National Accreditation Office in accordance with the requirements of ISO 17 025:2005 and ISO 15 189:2012, while the analysis of serum amino acids was done at Ansynth Service B.V., a laboratory specialising in amino acids (http://www. ansynth.com/). Serum aflatoxins and fumonisins were analysed at the Centre of Excellence in Mycotoxicology and Public Health, Faculty of Pharmaceutical Sciences, Ghent University, Belgium.

\section{LC-MS/MS analysis}

LC-MS/MS analyses were performed by Acquity ultra-highpressure liquid chromatography (UPLC) coupled to a Xevo TQ-S (Waters), equipped with a positive electrospray ionisation source (ESI). Two mobile phases were used: mobile phase A (95\% water and $5 \%$ methanol) and mobile phase B (95\% methanol and $5 \%$ water). Both phases were also adjusted with $5 \mathrm{mM}$ ammonium acetate and $0 \cdot 1 \%$ formic acid. The gradient elution programme started at 100\% mobile phase A. After an isocratic phase for $0.5 \mathrm{~min}$ at initial conditions, mobile phase B increased to $37 \%$ in $2.5 \mathrm{~min}$. Then, during a further $13 \mathrm{~min}$, phase $\mathrm{B}$ reached $75 \%$. Later, it was enhanced for $2 \mathrm{~min}$ with $100 \%$ mobile phase B. An equilibration step for 1.5 min was introduced, resulting in a total run time of $19.5 \mathrm{~min}$. The flow rate was set at $0.4 \mathrm{ml} / \mathrm{min}$. The mass spectrometer was operated in positive electrospray ionisation mode (ESI+). The capillary voltage was $30 \mathrm{kV}$, and nitrogen was applied as spray gas. The source and desolvation temperatures were set at $150{ }^{\circ} \mathrm{C}$ and $200^{\circ} \mathrm{C}$, respectively. The argon collision gas pressure was $9 \times 10^{-6} \mathrm{bar}$, the cone gas flow was $50 \mathrm{l} / \mathrm{h}$ and the desolvation gas flow was $500 \mathrm{l} / \mathrm{h}$ (see online supplementary material, Supplemental Table S1).

\section{Method validation}

The developed LC-MS/MS method was successfully validated based on the European Commission Decision 2002/657/EC, which provides rules for the analytical methods to be used to test official samples ${ }^{(32)}$. Matrix-matched calibration plots were constructed for the determination of the analytes. MassLynx $4 \cdot 1$ and TargetLynx $4 \cdot 1$ software (Micromass) were used for data acquisition and processing. The real or known compounds were identified using peak ratio (relative ion intensity), retention time and signal:noise ratio $^{(32)}$. After the completion of all criteria, the response, 
which is expressed as the ratio of the compound divided by the ratio of the area of the internal standard, was calculated.

Specificity was checked to ensure there was no interference or any peaks for the identification and quantification of the target compounds in the $\pm 2.5 \%$ margin of the relative time in five blank samples. Evaluating linearity, the homogeneity of variance was checked before fitting the linear model. Linearity was interpreted graphically using a scatter plot with the $r^{2}$ threshold set to $\geq 0.95$. Recovery was calculated after measuring the concentration and the actual (spiked) concentration. The observed concentration was calculated in triplicate from a matrix-matched calibration curve. The precision was calculated in terms of the intraday $\left(\mathrm{RSD}_{r}\right)$ and interday $\left(\mathrm{RSD}_{R}\right)$ precision. Limit of detection (LOD) was calculated as three times the standard error of the intercept, divided by the slope of the standard curve; the limit of quantification (LOQ) was similar, differing by six times the standard error. The calculated LOD and LOQ, which should be $>3$ and 10, respectively, were verified by the signal:noise ratio $(s / n)$, according to the International Union of Pure and Applied Chemistry guidelines. The results of performance characteristics of the LC-MS/MS method complied with the criteria outlined in European Commission Decision 2002/657/EC (see online supplementary material, Supplemental Table S2) ${ }^{(32)}$. Briefly, four identification points should be fulfilled to allow confirmation of the identity of the detected compound; one precursor and at least two product ions should be monitored; the relative intensities of the detected ions should correspond within accepted deviations to those of the calibration; detected ions should have a $s / n$ of at least 3 ; and the relative retention time of detected ions must range within a margin of $2.5 \%$.

\section{Statistical analyses}

As little information was available to hypothesise the prevalence of mycotoxin exposure in this population, a sample size of fifty children per stunting category was selected to achieve margins of error for $95 \%$ CIs of estimated proportions no greater than $\pm 14 \%$, with greater precision expected in estimates of weighted prevalence calculated from the full sample ( $n$ 102). The overall target sample size of 100 children provided $86 \%$ power to detect a correlation of 0.3 at 0.05 significance level, and statistical power was expected to increase with repeated measurement and adjustment for covariates in linear models. The final sample size was further constrained by the study budget. Exposure to any aflatoxins was defined as a binary variable indicating one or more aflatoxin biomarkers found at a detectable level in a child's serum. We used weighted prevalences of exposure, that is, adjusted for baseline stunting prevalence, to estimate aflatoxin and fumonisin exposure. To assess whether the exposure to aflatoxins was associated with linear growth, we measured height-for-age difference (HAD) and height-for-age $Z$-score (HAZ). HAD is preferred over HAZ when assessing changes in height/length-for-age in longitudinal data ${ }^{(33)}$. Stunting was defined as HAZ scores $<2$ SD below median values ${ }^{(30)}$. As part of the larger intervention study, households were grouped into wealth tertiles based on the first principal component constructed from household assets during the pre-harvest season, in particular agricultural transportation and household implements ${ }^{(34)}$.

Statistical analyses were conducted with SAS version 9.4 (SAS Institute), and all statistical tests used a 0.05 significance level. Wilcoxon tests were used to compare aflatoxin concentrations between children of different stunting status in each season. McNemar's tests using sampling weights were used to compare the prevalence of aflatoxin and fumonisin exposure across seasons. Spearman's rank correlation was used to investigate correlations among individual aflatoxins, inflammation (AGP and CRP), biomarkers of protein or amino acid status (serum transthyretin, IGF-1, lysine and tryptophan) and growth of children (HAZ and HAD). To examine these associations further, linear models accounting for repeated measures on children were fitted using the SAS procedure 'proc mixed' with restricted maximum likelihood estimation and the Kenward-Roger method to compute degree of freedom ${ }^{(35)}$. All regressions were repeated using sample weights; weighting did not materially change any of the study findings. Independent variables, common to all models, were those known or suspected to be important predictors of the outcomes: child's sex, age, time of assessment (pre- or post-harvest), intervention arm, inflammation (AGP and CRP, both natural log-transformed), household wealth tertile and incidence of diarrhoea in the preceding $14 \mathrm{~d}$. Model diagnostics were checked to ensure that assumptions of normality of error terms and homogeneity of error variance were met. For each outcome, each aflatoxin was considered individually as a predictor ('individual models'), and all aflatoxins were considered together as predictors in a 'joint model'. Further, using the joint model, a likelihood ratio test ('joint test') was conducted to test for the combined effect of all aflatoxins associated with the outcome (i.e. a statistical test of all aflatoxins as predictors $v$. none) $)^{(36)}$.

\section{Results}

At baseline (pre-harvest season), children were 21 months old on average and $65 \%$ were male. Fourteen per cent of mothers reported that their child had diarrheal symptoms in the 2 weeks prior to data collection. Stunted and non-stunted children did not differ significantly in age, sex, diarrheal incidence or household wealth (Table 1).

Half of the children (51\%) were exposed to some aflatoxins during the pre-harvest season (Fig. 2a). Exposure to any aflatoxin type was lower in the post-harvest season $(P<0.0001)$, but still remained high ( $41 \%)$. Across seasons, exposure was most prevalent for AFB1 (19-22\%), AFB2 
Table 1 Demographic and socioeconomic characteristics of study participants at baseline

\begin{tabular}{|c|c|c|c|c|c|c|c|}
\hline \multirow[b]{2}{*}{ Characteristics } & \multicolumn{4}{|c|}{ Growth status } & \multirow{2}{*}{\multicolumn{2}{|c|}{ All }} & \multirow[b]{2}{*}{$P$-value* } \\
\hline & \multicolumn{2}{|c|}{$\begin{array}{c}\text { Stunted } \\
(n 50)\end{array}$} & \multicolumn{2}{|c|}{$\begin{array}{l}\text { Non-stunted } \\
\quad(n 52)\end{array}$} & & & \\
\hline $\mathrm{HAZ}($ mean $\pm \mathrm{SD})$ & \multirow{3}{*}{\multicolumn{2}{|c|}{$\begin{array}{c}-2 \cdot 66 \pm 0.60 \\
-8 \cdot 00 \pm 2.29 \\
21.6 \pm 7.6\end{array}$}} & \multirow{3}{*}{\multicolumn{2}{|c|}{$\begin{aligned}-0.57 & \pm 0.95 \\
-1.80 & \pm 2.93 \\
19.8 & \pm 8.7\end{aligned}$}} & \multirow{3}{*}{\multicolumn{2}{|c|}{$\begin{array}{c}-1.59 \pm 1.32 \\
-4.84 \pm 4.08 \\
20.7 \pm 8.2\end{array}$}} & $<0.001$ \\
\hline $\mathrm{HAD}($ mean $\pm S D)$ & & & & & & & $<0.001$ \\
\hline \multirow[t]{2}{*}{ Age, months (mean $\pm S D$ ) } & & & & & & & 0.27 \\
\hline & $n$ & $\%$ & $n$ & $\%$ & $n$ & $\%$ & \\
\hline Sex (male) & 72 & 36 & 58 & 30 & 65 & 66 & 0.13 \\
\hline Presence of diarrheal illness in preceding 2 weeks ${ }^{3}$ & 12 & 6 & 15 & 8 & 14 & 14 & 0.62 \\
\hline \multicolumn{8}{|l|}{ Household wealth status ${ }^{4}$} \\
\hline Less poor & 16 & 23 & 34 & 17 & 25 & 41 & 0.12 \\
\hline Medium & 38 & 19 & 30 & 15 & 34 & 34 & \\
\hline Poorest & 46 & 8 & 36 & 18 & 41 & 25 & \\
\hline
\end{tabular}

HAZ: height-for-age Z-score; HAD: height-for-age difference.

*No weighting was used for $P$-values.

${ }^{3}$ Diarrheal illness was assessed by asking the mother whether in the preceding two weeks, the child had diarrhea (three or more loose or watery motions in a $24-\mathrm{h}$ period).

${ }^{4}$ As part of the larger intervention study, households were grouped into wealth tertiles based on the first principal component constructed from household assets during the preharvest season.

(a) Aflatoxins

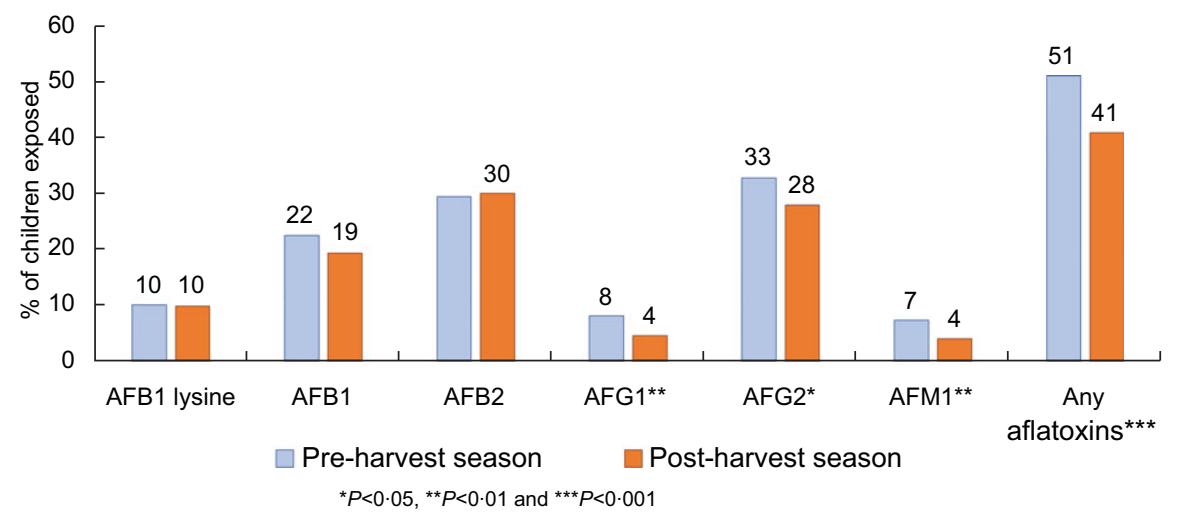

(b) Fumonisins

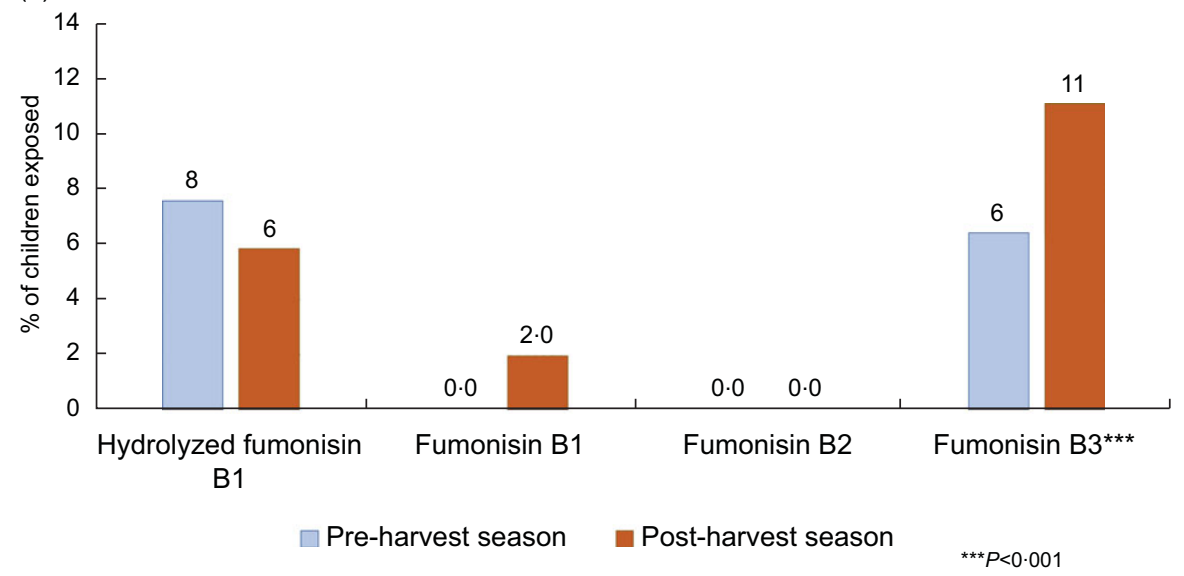

Fig. 2 Pre- and post-harvest weighted prevalence of exposure to aflatoxins (a) and fumonisins (b) as measured in serum

(29-30\%) and AFG2 (28-33\%); however, AFB1-Lys was low (10\%). The prevalence of AFB1-Lys, AFB1 and AFB2 in serum did not significantly change across seasons ( $P>0.05$ for each); however, AFG1 $(P=0.0048)$, AFG2 $(P=0.0192)$ and AFM1 $(P=0.0049)$ were all less prevalent in the post-harvest season, though prevalence of exposure to AFG2 remained high (28\%) (Fig. 2a). The proportion of children exposed to fumonisins was found to be low, ranging from $0 \%$ to $11 \%$ by type of fumonisin (Fig. 2b). Therefore, associations between exposure to fumonisins and the outcome variables were not further analysed in this study. 
Table 2 Upper percentiles* of aflatoxin concentrations measured in serum

\begin{tabular}{|c|c|c|c|c|c|c|c|c|}
\hline \multirow[b]{3}{*}{ Serum aflatoxin } & \multicolumn{4}{|c|}{ Pre-harvest (pg/ml) } & \multicolumn{4}{|c|}{ Post-harvest (pg/ml) } \\
\hline & \multicolumn{2}{|c|}{$\begin{array}{c}\text { Stunted } \dagger \\
(n 50)\end{array}$} & \multicolumn{2}{|c|}{$\begin{array}{c}\text { Not stunted } \dagger \\
\quad(n 52)\end{array}$} & \multicolumn{2}{|c|}{$\begin{array}{c}\text { Stunted } \dagger \\
(n 50)\end{array}$} & \multicolumn{2}{|c|}{$\begin{array}{l}\text { Not stunted } \dagger \\
\quad(n 52)\end{array}$} \\
\hline & $75 \%$ ile & Max & $75 \%$ ile & Max & $75 \%$ ile & Max & $75 \%$ ile & Max \\
\hline AFB1-Lys & $<$ LOD & $167 \cdot 0$ & $<L O D$ & $182 \cdot 0$ & $<$ LOD & $290 \cdot 0$ & $<$ LOD & $987 \cdot 6$ \\
\hline AFB1 & $<$ LOD & 18.5 & 8.400 & $19 \cdot 3$ & $<\mathrm{LOD}$ & 18.9 & $<\mathrm{LOD}$ & 21.0 \\
\hline AFB2 & $5 \cdot 700$ & $11 \cdot 3$ & $6 \cdot 000$ & $11 \cdot 3$ & $6 \cdot 500$ & 14.3 & $2 \cdot 700$ & $10 \cdot 9$ \\
\hline AFG1 & $<\mathrm{LOD}$ & $5 \cdot 6$ & $<\mathrm{LOD}$ & 5.9 & $<\mathrm{LOD}$ & $5 \cdot 8$ & $<\mathrm{LOD}$ & $6 \cdot 1$ \\
\hline AFG2 & 0.500 & $5 \cdot 6$ & 1.200 & 5.9 & 0.400 & $5 \cdot 1$ & 0.600 & $5 \cdot 6$ \\
\hline AFM1 & $<$ LOD & $11 \cdot 1$ & $<\mathrm{LOD}$ & $13 \cdot 2$ & $<\mathrm{LOD}$ & $10 \cdot 9$ & $<$ LOD & $10 \cdot 8$ \\
\hline
\end{tabular}

AFB1-Lys: aflatoxin B1-lysine; AFB1: aflatoxin B1; AFB2: aflatoxin B2; AFG1: aflatoxin G1; AFG2: aflatoxin G2; AFM1: aflatoxin M1.

${ }^{*}$ As all aflatoxins were found in $<50 \%$ of children, all median values were below the limit of detection (LOD).

$\dagger$ As measured at baseline.

Table 3 Correlations among aflatoxins and AFB1-Lys in each season

\begin{tabular}{|c|c|c|c|c|c|c|c|c|c|c|}
\hline \multicolumn{6}{|c|}{ Pre-harvest season } & \multicolumn{5}{|c|}{ Post-harvest season } \\
\hline & AFB1-Lys & AFB1 & AFB2 & AFG1 & AFG2 & AFB1-Lys & AFB1 & AFB2 & AFG1 & AFG2 \\
\hline AFB1 & 0.07 & & & & & $0 \cdot 20^{*}$ & & & & \\
\hline AFB2 & 0.01 & $0.63^{\star \star \star}$ & & & & 0.13 & $0.43^{\star \star \star}$ & & & \\
\hline AFG1 & -0.08 & -0.01 & $0.27^{\star \star}$ & & & 0.10 & $0.25^{\star}$ & 0.13 & & \\
\hline AFG2 & -0.004 & $0.41^{\star * \star}$ & $0.56^{\star \star \star}$ & $0.38^{\star * *}$ & & 0.19 & $0.49^{\star \star \star}$ & $0.65^{\star \star \star}$ & 0.12 & \\
\hline AFM1 & 0.04 & -0.13 & -0.07 & 0.09 & -0.08 & $0.27^{\star \star \star}$ & 0.04 & 0.12 & -0.05 & 0.05 \\
\hline
\end{tabular}

AFB1-Lys: aflatoxin B1-lysine; AFB1: aflatoxin B1; AFB2: aflatoxin B2; AFG1: aflatoxin G1; AFG2: aflatoxin G2; AFM1: aflatoxin M1.

Spearman's rank correlations, unweighted. Values below the limit of detection (LOD) were set to LOD/2.

${ }^{\star} P<0.05,{ }^{\star \star} P<0.01,{ }^{* \star *} P<0.001$.

The concentrations of individual aflatoxins varied in the serum of sampled children (Table 2). However, the concentrations did not differ $(P>0.05)$ by baseline stunting status at either time-point for any of the tested aflatoxins or AFB1-Lys (Table 2). AFB1-Lys was not correlated with any aflatoxins in the pre-harvest season; but in the post-harvest season, AFB1-Lys was positively correlated with AFB1 $(r=0.20, \quad P=0.04)$ and AFM1 $(r=0.27, \quad P=0.001)$ (Table 3$)$. AFB1 and AFB2 were positively correlated in both seasons $(P<0.001$ for both $)$ AFG1 was correlated with AFB2 $(r=0.27, P=0.005)$ in the pre-harvest season and with AFB1 $(r=0.25, P=0.01)$ in the post-harvest season, while AFG2 was positively correlated with AFB1 and AFB2 in both seasons ( $P<0.001$ for both).

The linear growth of children was not correlated with any aflatoxins in the pre-harvest season (all $P>0.05$ ) (see online supplementary material, Supplemental Table S3). Furthermore, none of the measured aflatoxins were correlated with a biomarker of protein in the pre-harvest season (see online supplementary material, Supplemental Table S3). AFB1 was inversely correlated with IGF-1 $(r=-0.26, P=0.009)$, and AFB2 was inversely correlated with serum tryptophan $(r=-0.33, P=0.001)$ in the post-harvest season (see online supplementary material, Supplemental Table S4).

Inflammation (AGP) was inversely correlated with serum biomarkers transthyretin $(r=-0.31, P=0.002)$, tryptophan $(r=-0 \cdot 23, P=0 \cdot 02)$ and IGF-1 $(r=-0 \cdot 25, P=0 \cdot 01)$ in the pre-harvest season (see online supplementary material, Supplemental Table S3). Similarly, it was inversely correlated with biomarkers transthyretin $(r=-0.39$, $P<0.0001)$ and tryptophan $(r=-0.25, P=0.012)$ in the post-harvest season (see online supplementary material, Supplemental Table S4).

HAZ in the pre-harvest season did not differ by exposure to aflatoxins in the same season $(P=0 \cdot 16$, Fig. 3a). Similarly, HAZ in the post-harvest season did not differ by aflatoxin exposure in the pre-harvest $(P=0 \cdot 14)$ or post-harvest seasons $(P=0 \cdot 15)$. Children exposed to any aflatoxin in either season also did not significantly differ in HAZ at the endline survey (see online supplementary material, Supplemental Table S5). However, when stunting (HAZ $<-2$ SD) was specifically considered, children exposed to aflatoxins in the pre-harvest season were less likely to be stunted in the same season $(P=0.01)$, contrary to expectation, though pre-harvest exposure was not associated with stunting in the post-harvest season $(P=0 \cdot 19)$ (Fig. 3b). In contrast, children exposed to aflatoxins in the post-harvest season were marginally more likely to be stunted in the same season $(P=0.08)$ (Fig. 3b). WHZ of the children was not correlated with exposure to aflatoxins in either season (see online supplementary material, Supplemental Table S3 and S4).

Exposure to any specific aflatoxin was not associated with acute or chronic inflammation (Table 4) or biomarkers of protein status (Table 5) in young children. However, AFB2 
exposure measured in serum was inversely associated with serum tryptophan $(P=0.0002)$ (Table 5$)$. Children's exposure to any specific aflatoxins was not associated with linear growth, as measured using HAZ or HAD (Table 6). However, all aflatoxins (AFB1, AFB2, AFG1, AFG2 and AFM1), when examined as a group using likelihood ratio tests ('joint tests' in Tables 4-6), had a significant association with each outcome (AGP, CRP, transthyretin, IGF-1, lysine, tryptophan, HAZ and HAD; $P<0.0001$ for each outcome except for transthyretin, for which $P=0.007$ ).
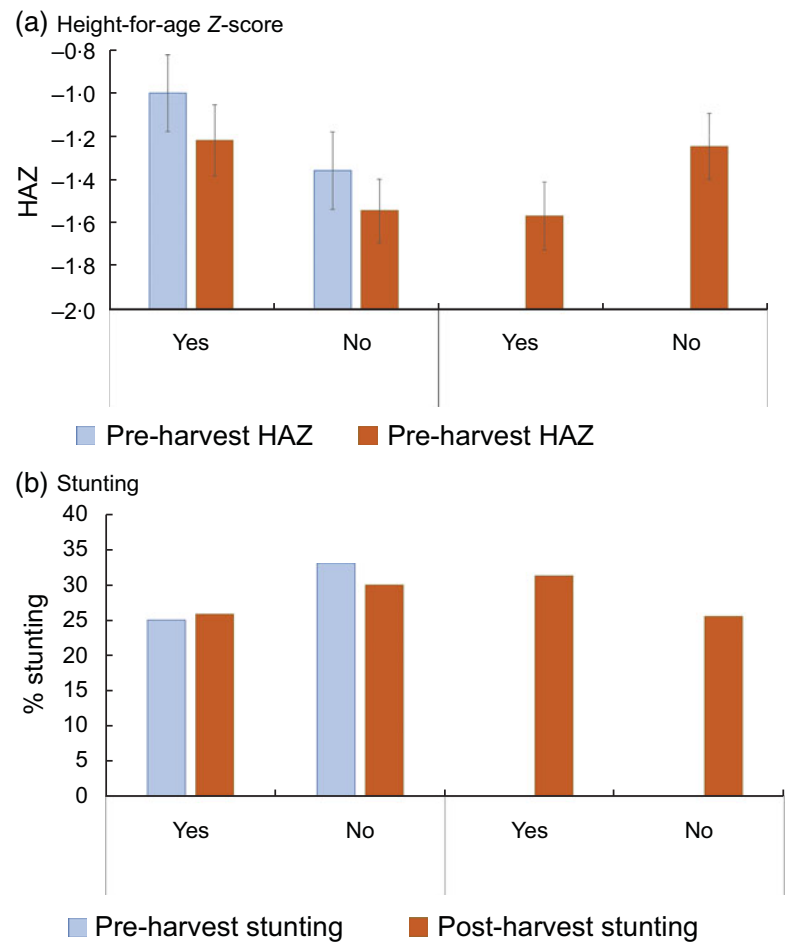

Fig. 3 Mean weighted height-for-age Z-scores (HAZ, panel a) and weighted prevalence of stunting (panel b) in pre- and post-harvest seasons, by exposure to any aflatoxins in either season

\section{Discussion}

We found a high prevalence of exposure to one or more aflatoxins in nearly half of our children during both preand post-harvest seasons. In our study population, all the carcinogenic aflatoxins could be detected in variable amounts. According to the International Agency for Research on Cancer, AFB1, AFB2, AFG1, AFG2 and AFM1 are highly carcinogenic for humans ${ }^{(9)}$. In general, the level of carcinogenicity is categorised in decreasing order as AFB1 > AFG1 > AFM1 > AFB2 > AFG2 ${ }^{(37)}$. Although exposure to aflatoxins in our population was lower than in some other African studies ${ }^{(18,23)}$, the current exposure data suggest that aflatoxin contamination is a public health problem in Ethiopia as zero tolerance for aflatoxin exposure is desirable. To our knowledge, this is the first study that assesses exposure to aflatoxins using multiple serum biomarkers, allowing us to compare the relative contribution of each to exposure risk. AFB1 is the most frequently reported carcinogen in many cereal-consuming populations $^{(38)}$. However, in our population, it was third highest, with one out of five children exposed to AFB1. The serum concentration of AFB1-Lys, an indicator of exposure over longer periods of time ${ }^{(38)}$, was not high, contrary to our expectation but comparable to some other studies ${ }^{(18,26)}$. AFB1-Lys in our studied population was much lower than the exposure found in several West African studies ${ }^{(17-22)}$. In our study, exposure to AFB2 and AFG2 was the most common, found in nearly a third of our study children. Interestingly, AFB2 and AFG2 are metabolites of AFB1 and AFG1, respectively ${ }^{(39)}$. The higher prevalence of $\mathrm{AFB} 2$ relative to $\mathrm{AFB} 1$, and especially $\mathrm{AFG} 2$ relative to AFG1, may be due to timing and duration of exposure. Also, the assays for AFB2 and AFG2 have lower LOD than the assays for AFB1 and AFG1, and concentrations measured were relatively low in our sample. These lower LOD may partially explain the higher prevalence detected for AFB2 and AFG2.

Table 4 Associations between aflatoxin exposures and inflammation among Ethiopian children*

\begin{tabular}{|c|c|c|c|c|c|c|c|c|c|c|c|c|}
\hline \multirow[b]{3}{*}{ Aflatoxin } & \multicolumn{6}{|c|}{ AGP } & \multicolumn{6}{|c|}{ CRP } \\
\hline & \multicolumn{3}{|c|}{ Individual models } & \multicolumn{3}{|c|}{ Joint model } & \multicolumn{3}{|c|}{ Individual models } & \multicolumn{3}{|c|}{ Joint model } \\
\hline & Estimate & SE & $P$-value & Estimate & SE & $P$-value & Estimate & SE & $P$-value & Estimate & SE & $P$-value \\
\hline AFB1-Lys & -0.59 & 0.68 & 0.39 & & & & 0.44 & 2.05 & 0.83 & & & \\
\hline AFB1 & -1.49 & 7.40 & 0.84 & $2 \cdot 72$ & $8 \cdot 14$ & 0.74 & $-17 \cdot 61$ & 21.91 & 0.42 & $-12 \cdot 20$ & 24.28 & 0.62 \\
\hline AFB2 & -17.54 & 10.53 & 0.10 & $-16 \cdot 08$ & $12 \cdot 52$ & 0.20 & -34.55 & $30 \cdot 60$ & 0.26 & -19.07 & 37.42 & 0.61 \\
\hline AFG1 & -4.93 & 33.99 & 0.88 & $13 \cdot 86$ & 34.93 & 0.69 & $-55 \cdot 37$ & $102 \cdot 21$ & 0.59 & -16.97 & $105 \cdot 23$ & 0.87 \\
\hline AFG2 & $-30 \cdot 82$ & $22 \cdot 27$ & 0.17 & -21.77 & 24.46 & 0.37 & $-56 \cdot 35$ & 62.47 & 0.37 & $-36 \cdot 95$ & 70.41 & 0.60 \\
\hline AFM1 & $-19 \cdot 38$ & $12 \cdot 14$ & 0.11 & $-19 \cdot 92$ & $12 \cdot 26$ & $0 \cdot 11$ & $-61 \cdot 44$ & 36.51 & 0.09 & $-62 \cdot 75$ & 36.96 & 0.09 \\
\hline Joint test & & & & & & $<0.0001$ & & & & & & $<0.0001$ \\
\hline
\end{tabular}

AFB1-Lys: aflatoxin B1-lysine; AFB1: aflatoxin B1; AFB2: aflatoxin B2; AFG1: aflatoxin G1; AFG2: aflatoxin G2; AFM1: aflatoxin M1; AGP: $\alpha$-1-glycoprotein; CRP: C-reactive protein.

*The quantitative effect of each aflatoxin or aflatoxin biomarker on AGP and CRP was assessed individually ('individual models') and together in a 'joint model'. All models controlled for child's sex, age, time of assessment (pre- or post-harvest) and intervention arm. In all models, the response variable (AGP or CRP) was natural log-transformed prior to analysis. Based on the joint model, the 'joint test' is a likelihood ratio test for the combined effect of all aflatoxins associated with the outcome (i.e. a statistical test of all aflatoxins as predictors $v$. none). 
Table 5 Associations between aflatoxin exposures and serum protein biomarkers among Ethiopian children*

\begin{tabular}{|c|c|c|c|c|c|c|c|c|c|c|c|c|}
\hline \multirow[b]{3}{*}{ Aflatoxin } & \multicolumn{6}{|c|}{ Transthyretin } & \multicolumn{6}{|c|}{ IGF-1* } \\
\hline & \multicolumn{3}{|c|}{ Individual models } & \multicolumn{3}{|c|}{ Joint model } & \multicolumn{3}{|c|}{ Individual models } & \multicolumn{3}{|c|}{ Joint model } \\
\hline & Estimate & SE & $P$-value & Estimate & SE & $P$-value & Estimate & SE & $P$-value & Estimate & SE & $P$-value \\
\hline \multirow{10}{*}{$\begin{array}{l}\text { AFB1-Lys } \\
\text { AFB1 } \\
\text { AFB2 } \\
\text { AFG1 } \\
\text { AFG2 } \\
\text { AFM1 } \\
\text { Joint test }\end{array}$} & 0.09 & 0.07 & 0.18 & & & & 0.69 & $1 \cdot 11$ & 0.54 & & & \\
\hline & -0.20 & 0.73 & 0.78 & 0.03 & 0.82 & 0.97 & -8.33 & 11.87 & 0.48 & -9.37 & 13.42 & 0.49 \\
\hline & -0.73 & 1.08 & 0.50 & -1.19 & 1.26 & 0.35 & 0.68 & $16 \cdot 77$ & 0.97 & 1.92 & 20.91 & 0.93 \\
\hline & -0.60 & 3.34 & 0.86 & -0.99 & 3.48 & 0.78 & 0.15 & $58 \cdot 01$ & 1.00 & -3.86 & 59.96 & 0.95 \\
\hline & $2 \cdot 29$ & $2 \cdot 38$ & 0.34 & $3 \cdot 22$ & 2.59 & 0.21 & 19.88 & 32.78 & 0.55 & 21.09 & 37.79 & 0.58 \\
\hline & -0.56 & $1 \cdot 22$ & 0.65 & -0.49 & $1 \cdot 23$ & 0.69 & $4 \cdot 32$ & $20 \cdot 24$ & $0 \cdot 83$ & 3.80 & $20 \cdot 52$ & 0.85 \\
\hline & & & & & & 0.007 & & & & & & $<0.0001$ \\
\hline & \multicolumn{6}{|c|}{ Lysine } & \multicolumn{6}{|c|}{ Tryptophan } \\
\hline & \multicolumn{3}{|c|}{ Individual models } & \multicolumn{3}{|c|}{ Joint model } & \multicolumn{3}{|c|}{ Individual models } & \multicolumn{3}{|c|}{ Joint model } \\
\hline & Estimate & SE & $P$-value & Estimate & SE & $P$-value & Estimate & SE & $P$-value & Estimate & SE & $P$-value \\
\hline AFB1-Lys & -70.07 & 64.44 & 0.28 & & & & $-10 \cdot 25$ & $25 \cdot 90$ & 0.69 & & & \\
\hline AFB1 & -1191.01 & 688.09 & 0.09 & -772.05 & 761.91 & 0.31 & $358 \cdot 39$ & $276 \cdot 33$ & 0.20 & 835.41 & 297.83 & 0.006 \\
\hline AFB2 & $-1540 \cdot 33$ & 977.46 & 0.12 & -1574.30 & 1178.51 & 0.18 & $-1191 \cdot 73$ & 388.74 & 0.003 & $-1762 \cdot 41$ & $460 \cdot 30$ & 0.0002 \\
\hline AFG1 & 2247.66 & 3217.94 & 0.49 & 2583.35 & 3300.23 & 0.43 & -158.25 & 1284.54 & 0.90 & 408.98 & 1284.44 & 0.75 \\
\hline AFG2 & $606 \cdot 54$ & 2014.48 & 0.76 & 1923.96 & $2220 \cdot 26$ & 0.39 & -835.06 & $825 \cdot 50$ & 0.31 & 298.95 & $881 \cdot 82$ & 0.74 \\
\hline $\begin{array}{l}\text { AFM1 } \\
\text { Joint test }\end{array}$ & $1709 \cdot 14$ & $1159 \cdot 02$ & $0 \cdot 14$ & $1525 \cdot 81$ & $1168 \cdot 37$ & $\begin{aligned} & 0.19 \\
< & 0.0001\end{aligned}$ & $-228 \cdot 19$ & $466 \cdot 23$ & 0.63 & $-187 \cdot 73$ & $454 \cdot 82$ & $\begin{aligned} & 0.68 \\
< & 0.0001\end{aligned}$ \\
\hline
\end{tabular}

AFB1-Lys: aflatoxin B1-lysine; AFB1: aflatoxin B1; AFB2: aflatoxin B2; AFG1: aflatoxin G1; AFG2: aflatoxin G2; AFM1: aflatoxin M1.

Response variable was natural log-transformed prior to analysis.

'The quantitative effect of each aflatoxin or aflatoxin biomarker on serum biomarkers was assessed individually ('individual models') and together in a 'joint model'. All models controlled for child's sex, age, time of assessment (pre- or postharvest), intervention arm and inflammation (AGP and CRP, both natural log-transformed). Based on the joint model, the 'joint test' is a likelihood ratio test for the combined effect of all aflatoxins associated with the outcome (i.e. a statistical test of all aflatoxins as predictors $V$. none) 
Table 6 Associations between aflatoxin exposures and linear growth among Ethiopian children*

\begin{tabular}{|c|c|c|c|c|c|c|c|c|c|c|c|c|}
\hline \multirow[b]{3}{*}{ Aflatoxin } & \multicolumn{6}{|c|}{ Height-for-age Z-score } & \multicolumn{6}{|c|}{ Height-for-age difference } \\
\hline & \multicolumn{3}{|c|}{ Individual models } & \multicolumn{3}{|c|}{ Joint model } & \multicolumn{3}{|c|}{ Individual models } & \multicolumn{3}{|c|}{ Joint model } \\
\hline & Estimate & SE & $P$-value & Estimate & SE & $P$-value & Estimate & SE & $P$-value & Estimate & SE & $P$-value \\
\hline AFB1-Lys & $2 \cdot 25$ & 1.50 & 0.14 & & & & 6.04 & 4.52 & 0.18 & & & \\
\hline AFB1 & 8.91 & $16 \cdot 76$ & 0.60 & $20 \cdot 30$ & $18 \cdot 76$ & 0.28 & -11.53 & 50.57 & 0.82 & 36.56 & $56 \cdot 47$ & 0.52 \\
\hline AFB2 & $-12 \cdot 60$ & $25 \cdot 31$ & 0.62 & -21.49 & 28.60 & 0.45 & -99.00 & 76.06 & 0.20 & -109.54 & 85.95 & 0.21 \\
\hline AFG1 & $24 \cdot 63$ & 73.13 & 0.74 & $41 \cdot 36$ & $76 \cdot 68$ & 0.59 & $37 \cdot 27$ & $219 \cdot 87$ & 0.87 & $115 \cdot 77$ & $230 \cdot 14$ & 0.62 \\
\hline AFG2 & -58.37 & 63.15 & 0.36 & -63.72 & $67 \cdot 13$ & 0.34 & $-225 \cdot 68$ & 193.63 & 0.25 & $-206 \cdot 90$ & $205 \cdot 74$ & 0.32 \\
\hline AFM1 & 46.99 & $26 \cdot 32$ & 0.08 & $46 \cdot 79$ & $26 \cdot 79$ & 0.08 & $143 \cdot 76$ & $79 \cdot 10$ & 0.07 & $137 \cdot 81$ & $80 \cdot 23$ & 0.09 \\
\hline
\end{tabular}

AFB1-Lys: aflatoxin B1-lysine; AFB1: aflatoxin B1; AFB2: aflatoxin B2; AFG1: aflatoxin G1; AFG2: aflatoxin G2; AFM1: aflatoxin M1.

*The quantitative effect of each aflatoxin or aflatoxin biomarker on indicators of linear growth was assessed individually ('individual models') and together in a 'joint model'. All models controlled for child's sex, age, time of assessment (pre- or post-harvest), intervention arm, inflammation (AGP and CRP, both natural log-transformed), household wealth tertile and incidence of diarrhoea in the preceding $14 \mathrm{~d}$. Based on the joint model, the 'joint test' is a likelihood ratio test for the combined effect of all aflatoxins associated with the outcome (i.e. a statistical test of all aflatoxins as predictors $v$. none).

The prevalence of exposure to AFM1, a metabolite found in milk and milk products when animals are fed contaminated feeds, was low in our study. An experimental study of animals showed that both AFB1 and AFM1 were detected in the plasma of cows after the ingestion of AFB1 on corn-based feed ${ }^{(40)}$. As reported in our previous paper ${ }^{(29)}$, milk consumption among children in our study area was minimal, which probably led to low levels of AFM1 contamination. AFG2 was quantitatively the most important type of aflatoxin, detected in about a third of our population. AFG1, on the other hand, was present in only a few samples, although both AFG1 and AFG2 are metabolites of $\mathrm{AFB} 1^{(40)}$.

Exposure to AFG1, AFG2 and AFM1 was higher in the pre-harvest season than in the post-harvest season. This could have been due to several reasons. In the pre-harvest season, complementary foods consumed by children were mostly prepared from foods produced and stored on household farms. The higher prevalence of aflatoxins detected in the pre-harvest season could have been caused by poor storage conditions over a long period of time, leading to fungal proliferation and mycotoxin contamination of grain used to prepare complementary foods. Research on the sources of mycotoxin exposure, including the effects of crop storage technologies, practices and duration on mycotoxin contamination, is needed to develop effective mitigation strategies.

Exposure to fumonisins was low in our study population. To our knowledge, exposure to fumonisins has never been measured before in human serum. An in vitro study showed that fumonisins might have the potential to cause severe hepatotoxic, nephrotoxic, hepatocarcinogenic and other cytotoxic effects in mammals ${ }^{(41)}$. Fumonisin B1 is the most toxic fumonisin ${ }^{(37)}$, but a very low fumonisin B1 exposure (2\%) was found in the post-harvest season and was not detectable in the pre-harvest season. Fumonisins are the second most important mycotoxins found on maize and in a variety of maize-based human foods, particularly when grown in warmer regions ${ }^{(42,43)}$. Fumonisin exposure from maize and other cereal-based foods has been reported in many developing countries ${ }^{(44)}$ but not in serum. The low level of exposure to fumonisins in our population might be because fumonisins are poorly absorbed and are excreted largely in faeces ${ }^{(39)}$. The other reason could be a low level of contamination in complementary foods in our study area. Despite the low fumonisin levels detected in this study, the authors strongly suggest continuing to study fumonisin exposure in Ethiopia as our study was conducted in only a small sample not representative of the entire country. However, future studies should take other matrixes, such as faeces or hair, to assess better fumonisin exposure ${ }^{(39)}$.

Exposure to AFB1 was inversely correlated with serum IGF-1, while AFB2 exposure was inversely correlated with serum tryptophan in the post-harvest season. However, the correlation between AFB1 and IGF-1 disappeared in regression models adjusted for confounders. Exposure to individual aflatoxins, including the AFB1-Lys biomarker, in serum was not associated with linear growth of children after adjusting for confounders. While individual aflatoxins largely did not have significant associations with inflammation, protein status or linear growth, they did have significant associations as a group to these outcomes, as evidenced by the joint tests. These relationships should be investigated further in larger studies that would have more statistical power.

Similar to our study, studies from other East African countries ${ }^{(23-25)}$ and $\mathrm{Nepal}^{(26)}$ did not find an association between AFB1-Lys and linear growth of children. Some observational and cross-sectional studies in West Africa found an inverse relationship between AFB1-Lys biomarkers and child growth ${ }^{(17,19-22)}$. Similarly, an observational study from Mexico showed that the level of exposure to aflatoxins was inversely associated with linear growth ${ }^{(18)}$. A recent randomised control trial among Kenyan children found that in households that had access to aflatoxin-free maize, children had reduced serum aflatoxin concentrations, but the linear growth of children was not affected ${ }^{(23)}$. 
There are a number of differences between our study and previous studies, most notably in the methods of aflatoxin analyses and study setting. The LC-MS/MS method used in our analyses is a more specific and sensitive technique ${ }^{(22)}$ compared with ELISA ${ }^{(45)}$. Also, most prior studies measured a single aflatoxin as a marker of exposure. The large variability in aflatoxins in serum and in linear growth suggests that further studies with larger sample sizes may be required to detect associations or causal linkages between aflatoxin exposure and growth of children. Even without a biological mechanism linking aflatoxin exposure and linear growth faltering, aflatoxin exposure remains a critical concern, given its carcinogenicity and other potential implications for health as well as international trade ${ }^{(46)}$.

When trying to elucidate factors that could explain a relationship between exposure to aflatoxins and growth, Smith and colleagues hypothesised that aflatoxin exposure may inhibit protein synthesis and increase inflammation or infection $^{(27)}$. We therefore investigated this hypothesis but found an unclear association between exposure to the group of aflatoxins and inflammation or protein status biomarkers such as serum transthyretin. Serum concentrations of tryptophan and lysine may mediate protein synthesis because these are the two most limiting amino acids in maize-based diets. Exposure to the group of aflatoxins was associated with these serum amino acids; however, only AFB1 and AFB2 were specifically associated with serum tryptophan.

In our study, the unclear association between aflatoxin biomarkers in serum and selected biomarkers of protein synthesis may have been due to relatively low serum concentrations of aflatoxin biomarkers, even though the prevalence of exposure was high. These associations between aflatoxin exposure and biomarkers of protein synthesis and inflammation may suggest the need for further study to better understand the biological mechanisms that could also lead to linear growth faltering in children.

Small sample size was a limitation in our study since aflatoxins could only be assessed for a subset of 102 children, meaning that our study may have been underpowered to detect smaller effects from low-level aflatoxin exposure. Further, the study did not take samples of children's food to analyse aflatoxin levels, which would have shed light on the origin of aflatoxins found in children's serum. Another limitation was the use of observational (nonexperimental) data, which does not allow us to infer causality. However, experimental investigation on the health effects of aflatoxin exposure in children would be ethically challenging at best, and our analyses adjusted for key known confounders. The high levels of aflatoxin exposure warrant further research to identify the sources of exposure and interventions to mitigate that exposure. These findings also motivate more widespread monitoring of mycotoxin exposure in Ethiopia, with attention to the possibility that exposure may vary over time.
We conclude that children in rural Ethiopia are widely exposed to aflatoxins, which can have both acute and long-term consequences for health, growth and development. However, the biological mechanisms by which aflatoxins may specifically affect inflammation, protein status or linear growth did not become clear from our study. A larger longitudinal study is therefore recommended to evaluate causal linkages between aflatoxin exposure and key nutrition and health outcomes in children.

\section{Acknowledgements}

Acknowledgements: The authors would like to thank Karl De Ruyck and Prof. Sarah De Saeger for supervising the analyses of serum mycotoxins, Dr David Miller and Dr Mark Sumarah for supplying the mycotoxin standards. We would also like to thank Liz Lucas for English editing the manuscript. Financial support: This work was funded by the Agricultural Technology Adoption Initiative grant OPP52811 from the Bill and Melinda Gate Foundation to the Harvard T.H. Chan School of Public Health, Boston, USA and the Nutritious Maize of Ethiopia Project. Conflict of interest: The authors have declared that no competing interests exist. Authorship: MT, NSG, IDB, HDG, TB, EJM and BJS conceived the study design; MT, AK, MDB and AVC performed the experiments; MT, NSG, HDG and IDB analysed the data; MT, HDG, IDB and NSG wrote up the first draft of the manuscript; and the manuscript was critically reviewed and refined by all authors. Ethics of human subject participation: The study was conducted according to the guidelines laid down in the Declaration of Helsinki, and all procedures involving data collection from human subjects were approved by the Ethiopian Public Health Institute Scientific and Ethical Review Committee (SERO006-02-2015). Prior to any data collection, the research staff obtained written informed consent from the parents of children who were interviewed, specifically the household head and caregiver.

\section{Supplementary material}

For supplementary material accompanying this paper visit https://doi.org/10.1017/S1368980021000422

\section{References}

1. Victora CG, Adair L, Fall C et al. (2008) Maternal and child undernutrition: consequences for adult health and human capital. Lancet 371, 340-357.

2. Murray CJL (2015) Shifting to sustainable development goals: implications for global health. N Engl J Med 373, 1390-1393.

3. WHO (2014) Global Nutrition Targets 2025: Stunting Policy Brief (WHO/NMH/NHD/14.3). Geneva: WHO. 
4. de Onis M, Dewey KG, Borghi E et al. (2013) The World Health Organization's global target for reducing childhood stunting by 2025: rationale and proposed actions. Matern Child Nutr 9, 6-26.

5. Millward DJ (2017) Nutrition, infection and stunting: the roles of deficiencies of individual nutrients and foods, and of inflammation, as determinants of reduced linear growth of children. Nutr Res Rev 30, 50-72.

6. Dewey KG \& Adu-Afarwuah S (2008) Systematic review of the efficacy and effectiveness of complementary feeding interventions in developing countries. Matern Child Nutr 4, 24-85.

7. Williams JH, Phillips TD, Jolly PE et al. (2004) Human aflatoxicosis in developing countries: a review of toxicology, exposure, potential health consequences, and interventions. Am J Clin Nutr 80, 1106-1122.

8. Wild CP \& Gong YY (2009) Mycotoxins and human disease: a largely ignored global health issue. Carcinog 31, 71-82.

9. International Agency for Research on Cancer (2015) Mycotoxin Control in Low- and Middle-Income Countries. Lyon: International Agency for Research on Cancer.

10. Samapundo S, Devlieghere F, Geeraerd A et al. (2007) Modelling of the individual and combined effects of water activity and temperature on the radial growth of Aspergillus flavus and A. parasiticus on corn. Food Microbiol 24, 517-529.

11. Creppy EE (2002) Update of survey, regulation and toxic effects of mycotoxins in Europe. Toxicol Lett 127, 19-28.

12. Torre LA, Siegel RL, Ward EM et al. (2016) Global cancer incidence and mortality rates and trends - an update. Cancer Epidemiol Biomarkers Prev 25, 16-27.

13. Magoha H, Kimanya M, De Meulenaer B et al. (2016) Risk of dietary exposure to aflatoxins and fumonisins in infants less than $6 \mathrm{~m}$ of age in Rombo, Northern Tanzania. Matern Child Nutr 12, 516-527.

14. Mwalwayo DS \& Thole B (2016) Prevalence of aflatoxin and fumonisins $(\mathrm{B} 1+\mathrm{B} 2)$ in maize consumed in rural Malawi. Toxicol Rep 3, 173-179.

15. Kimanya ME, De Meulenaer B, Tiisekwa B et al. (2008) Co-occurrence of fumonisins with aflatoxins in home-stored maize for human consumption in rural villages of Tanzania. Food Addit Contam Part A Chem Anal Control Expo Risk Assess 25, 1353-1364.

16. Kimanya ME, Shirima CP, Magoha H et al. (2014) Co-exposures of aflatoxins with deoxynivalenol, fumonisins from maize based complementary foods in Rombo, Northern Tanzania. Food Control 41, 76-81.

17. Watson S, Moore SE, Darboe MK et al. (2018) Impaired growth in rural Gambian infants exposed to aflatoxin: a prospective cohort study. BMC Public Health 18, 1247.

18. Leroy JL, Sununtnasuk C, Garcia-Guerra A et al. (2018) Low level aflatoxin exposure associated with greater linear growth in southern Mexico: a longitudinal study. Matern Child Nutr 14, e12619.

19. Turner PC, Collinson AC, Cheung YB et al. (2007) Aflatoxin exposure in utero causes growth faltering in Gambian infants. Int J Epidemiol 36, 1119-1125.

20. Gong Y, Hounsa A, Egal S et al. (2004) Postweaning exposure to aflatoxin results in impaired child growth: a longitudinal study in Benin, West Africa. Environ Health Perspect 112, 1334.

21. Gong YY, Cardwell K, Hounsa A et al. (2002) Dietary aflatoxin exposure and impaired growth in young children from Benin and Togo: cross sectional study. BMJ 325, 20-21.

22. McMillan A, Renaud JB, Burgess KM et al. (2018) Aflatoxin exposure in Nigerian children with severe acute malnutrition. Food Chem Toxicol 111, 356-362.

23. Hoffmann V, Jones K \& Leroy JL (2018) The impact of reducing dietary aflatoxin exposure on child linear growth: a cluster randomised controlled trial in Kenya. BMJ Global Health 3, e000983.

24. Shirima CP, Kimanya ME, Routledge MN et al. (2014) A prospective study of growth and biomarkers of exposure to aflatoxin and fumonisin during early childhood in Tanzania. Environ Health Perspect 123, 173-178.

25. Chen C, Mitchell NJ, Gratz J et al. (2018) Exposure to aflatoxin and fumonisin in children at risk for growth impairment in rural Tanzania. Environ Int 115, 29-37.

26. Mitchell NJ, Hsu H-H, Chandyo RK et al. (2017) Aflatoxin exposure during the first 36 months of life was not associated with impaired growth in Nepalese children: an extension of the MAL-ED study. PLoS One 12, e0172124.

27. Smith LE, Stoltzfus RJ \& Prendergast A (2012) Food chain mycotoxin exposure, gut health, and impaired growth: a conceptual framework. Adv Nutr: Int Rev J 3, 526-531.

28. Tessema M, Gunaratna NS, Donato K et al. (2016) Translating the impact of quality protein maize into improved nutritional status for Ethiopian children: study protocol for a randomized controlled trial. BMC Nutr $\mathbf{2}, 54$.

29. Tessema M, Gunaratna N, Brouwer I et al. (2018) Associations among high-quality protein and energy intake, serum transthyretin, serum amino acids and linear growth of children in Ethiopia. Nutrients 10, 1776.

30. World Health Organization (2008) Child Growth Standards 2006. Geneva: WHO.

31. Evans AM, DeHaven CD, Barrett T et al. (2009) Integrated, nontargeted ultrahigh performance liquid chromatography/electrospray ionization tandem mass spectrometry platform for the identification and relative quantification of the small-molecule complement of biological systems. Anal Chem 81, 6656-6667.

32. Commission of the European Communities (2002) Commission Decision 2002/657/EC implementing Council Directive 96/23/EC concerning the performance of analytical methods and the interpretation of results. OJ $L \mathbf{2 2 1}, 8$.

33. Leroy JL, Ruel M, Habicht J-P et al. (2015) Using heightfor-age differences (HAD) instead of height-for-age z-scores (HAZ) for the meaningful measurement of population-level catch-up in linear growth in children less than 5 years of age. BMC Pediatr 15, 145.

34. Filmer D \& Pritchett LH (2001) Estimating wealth effects without expenditure data - or tears: an application to educational enrollments in states of India. Demography 38, 115-132.

35. Kenward MG \& Roger JH (1997) Small sample inference for fixed effects from restricted maximum likelihood. Biometrics 53, 983-997.

36. Casella G \& Berger RL (2002) Statistical Inference, Duxbury/ Thomson Learning. Available at: https://fsalamri.files. wordpress.com/2015/02/casella_berger_statistical_inference1. pdf (accessed June 2019).

37. International Agency for Research on Cancer (1993) Some Naturally Occurring Substances: Food Items and Constituents, Heterocyclic Aromatic Amines and Mycotoxins. IARC Monographs Evaluation of the Carcinogenic Risk Chemicals to Humans. Geneva: World Health Organization.

38. Khlangwiset P, Shephard GS \& Wu F (2011) Aflatoxins and growth impairment: a review. Crit Rev Toxicol 41, 740-755.

39. Vidal A, Mengelers M, Yang S et al. (2018) Mycotoxin biomarkers of exposure: a comprehensive review. Compr Rev Food Sci Food Saf 17, 1127-1155.

40. Gallo A, Moschini M \& Masoero F (2008) Aflatoxins absorption in the gastro-intestinal tract and in the vaginal mucosa in lactating dairy cows. Ital J Anim Sci 7, 53-63.

41. Norred WP, Wang E, Yoo $\mathrm{H}$ et al. (1992) In vitro toxicology of fumonisins and the mechanistic implications. Mycopathologia 117, 73-78. 
42. Marin S, Ramos A, Cano-Sancho G et al. (2013) Mycotoxins: occurrence, toxicology, and exposure assessment. Food Chem Toxicol 60, 218-237.

43. James A \& Zikankuba VL (2018) Mycotoxins contamination in maize alarms food safety in sub-Sahara Africa. Food Control 90, 372-381.

44. Carballo D, Tolosa J, Ferrer E et al. (2019) Dietary exposure assessment to mycotoxins through total diet studies. A review. Food Chem Toxicol 128, 8-20.
45. Wild CP, Hudson GJ, Sabbioni G et al. (1992) Dietary intake of aflatoxins and the level of albumin-bound aflatoxin in peripheral blood in The Gambia, West Africa. Cancer Epidemiol Prev Biomarkers 1, 229-234.

46. Leroy JL \& Frongillo EA (2019) Perspective: what does stunting really mean? a critical review of the evidence. Adv Nutr 10, 196-204. 